\title{
THE LESSON LEARNT DURING INTERACT - I AND INTERACT - II ACTRIS MEASUREMENT CAMPAIGNS
}

\author{
Marco Rosoldi ${ }^{1 *}$, Fabio Madonna ${ }^{1}$, Gelsomina Pappalardo ${ }^{1}$, Joshua Vande Hey ${ }^{2}$, Yunhui Zheng ${ }^{3}$, \\ VAISALA Team ${ }^{4}$ \\ ${ }^{1}$ Consiglio Nazionale delle Ricerche, Istituto di Metodologie per l'Analisi Ambientale (CNR-IMAA), Italy, \\ *marco.rosoldi@imaa.cnr.it \\ ${ }^{2}$ University of Leicester, Department of Physics and Astronomy, Earth Observation Science Group, UK \\ ${ }^{3}$ Sigma Space Corporation, USA \\ ${ }^{4}$ VAISALA, Finland
}

\begin{abstract}
The INTERACT-II (INTERcomparison of Aerosol and Cloud Tracking) campaign, performed at the CNR-IMAA Atmospheric Observatory $\left(760 \mathrm{~m}\right.$ a.s.1., $\left.40.60^{\circ} \mathrm{N}, 15.72^{\circ} \mathrm{E}\right)$, aims to evaluate the performances of commercial automatic lidars and ceilometers for atmospheric aerosol profiling, through the comparison with Potenza EARLINET (European Aerosol Research Lidar NETwork) lidars. The results of the campaign and the overall lesson learnt within INTERACT-I and INTERACT-II ACTRIS campaigns will be presented.
\end{abstract}

\section{INTRODUCTION}

Knowledge of aerosol spatio-temporal distribution in troposphere is essential to improve our understanding of climate and air quality. For this purpose, global scale high resolution continuous measurements of tropospheric aerosols are needed. Global coverage high resolution networks of ground-based low-cost and low-maintenance remote sensing instruments, such as commercial automatic lidars and ceilometers, can strongly contribute to this scientific mission. Therefore, it is very interesting for scientific community to understand to which extent these instruments are able to provide reliable aerosol measurements and fill in the geographical gaps of existing networks of advanced lidars, like EARLINET (European Aerosol Research LIdar NETwork). The INTERACT-II (INTERcomparison of Aerosol and Cloud Tracking) campaign, carried out at CIAO (CNR-IMAA Atmospheric Observatory) in Tito Scalo, Potenza, Italy (760 m a.s.1., $40.60^{\circ} \mathrm{N}$, $15.72^{\circ} \mathrm{E}$ ), aims to evaluate the performances of commercial automatic lidars and ceilometers for tropospheric aerosol profiling. The campaign has been performed in the period from July 2016 to January 2017 in the framework of ACTRIS-2 (Aerosol Clouds Trace gases Research InfraStructure) H2020 research infrastructure project. Besides the commercial ceilometers operational at CIAO, a Vaisala CT25K and a Luftt CHM15k (not operated from July to September due to the misalignment between laser and detector), the performance of a CL51 Vaisala ceilometer, a Campbell CS135 ceilometer and a Sigma Space mini-Micro Pulse Lidar (MPL) are assessed using the EARLINET multi-wavelength Raman lidars operative at CIAO as reference. In the following sections, the methodology adopted for the intercomparison is described along with the first results.

\section{METHODOLOGY}

The signals of the multi-wavelength Raman lidar operative at CIAO, MUSA, are processed using the EARLINET Single Calculus Chain (SCC) also with the aim to improve the data consistency and comparability $[1,2]$. The SCC provides the preprocessed range corrected signals (RCS) and the profiles of aerosol extinction coefficient at 355 and $532 \mathrm{~nm}$ and backscattering coefficient at 355 , 532 and $1064 \mathrm{~nm}$, using both Raman and elastic signals. MUSA is equipped with two channels detecting the co- and cross polarized components of the elastically backscattered radiation at 532 $\mathrm{nm}$, in order to measure the particle depolarization at that wavelength. The automatic mini-MPL detects the co- and cross polarized components of the elastically backscattered radiation at $532 \mathrm{~nm}$ and provides continuous measurements of particle backscattering coefficient and depolarization ratio profiles. In a first stage, the comparison between MUSA and mini-MPL is focused on the signals collected by both the instruments. Because of the 
different polarization setup of MUSA [3] and mini-MPL [4], total signals calculated from the combination of the respective co- and crosspolarized channels are compared. In particular, RCS are compared, which are proportional to attenuated backscatter coefficient profiles used in the first INTERACT campaign for the comparison of ceilometers and MUSA [5]. Mini-MPL RCS is normalized on the MUSA RCS in a vertical range of about $1.5 \mathrm{~km}$ between 8 and $10 \mathrm{~km}$ above sea level (a.s.l.), identified as an aerosol free region from the quicklooks of the lidar time series.

The performances of CL51 and CS135 are assessed by comparing their attenuated backscatter coefficient profiles with those of MUSA, following a similar approach used in the first INTERACT campaign. To compare attenuated backscatter coefficient profiles at 905 and $1064 \mathrm{~nm}$, obtained from the ceilometers and MUSA respectively, the spectral dependency of the attenuated backscatter coefficient is considered, using the backscatter related Angstrom exponent at 1064-532 nm retrieved from the processing of MUSA measurements. This is assumed to be the best approximation of the 1064-905 nm backscatter-related Angstrom exponent. Since ceilometers are characterized by a low signal to noise ratio (SNR) in the upper troposphere, their attenuated backscatter coefficient profiles are normalized on the corresponding MUSA profiles in the lower troposphere, in order to obtain a stable normalization. Typically, CL51 profiles are normalized in a vertical region between 4 and 5 $\mathrm{km}$ a.s.l.

All the time series considered in this comparison refer to night time measurements. The profiles from all the instruments are compared over a vertical resolution of 60 meters and a temporal integration ranging between 1 and 2 hours, depending on the observed atmospheric scenario. No vertical smoothing is applied to the data processing and all the profiles are cut in lower part of the atmosphere, below $1300 \mathrm{~m}$ a.s.l., to remove overlap effects.

A typical comparison between RCS provided by MUSA and mini-MPL is shown in the top panel of Figure 1. It is related to the observations collected on 29 August 2016 from 19:16 to 20:47 UT. The quicklooks of RCS time series (not reported) show a thick aerosol layer between 2 and $3 \mathrm{~km}$ a.s.l. The comparison shows a good agreement in the detection of the vertical structure of the atmospheric aerosol, though above $1.7 \mathrm{~km}$ a.s.l. a sort of bias affects most of the mini-MPL profile. This bias could be due to wrong corrections applied in the mini-MPL signal preprocessing, such as pile-up or trigger delay. Further investigation is required.
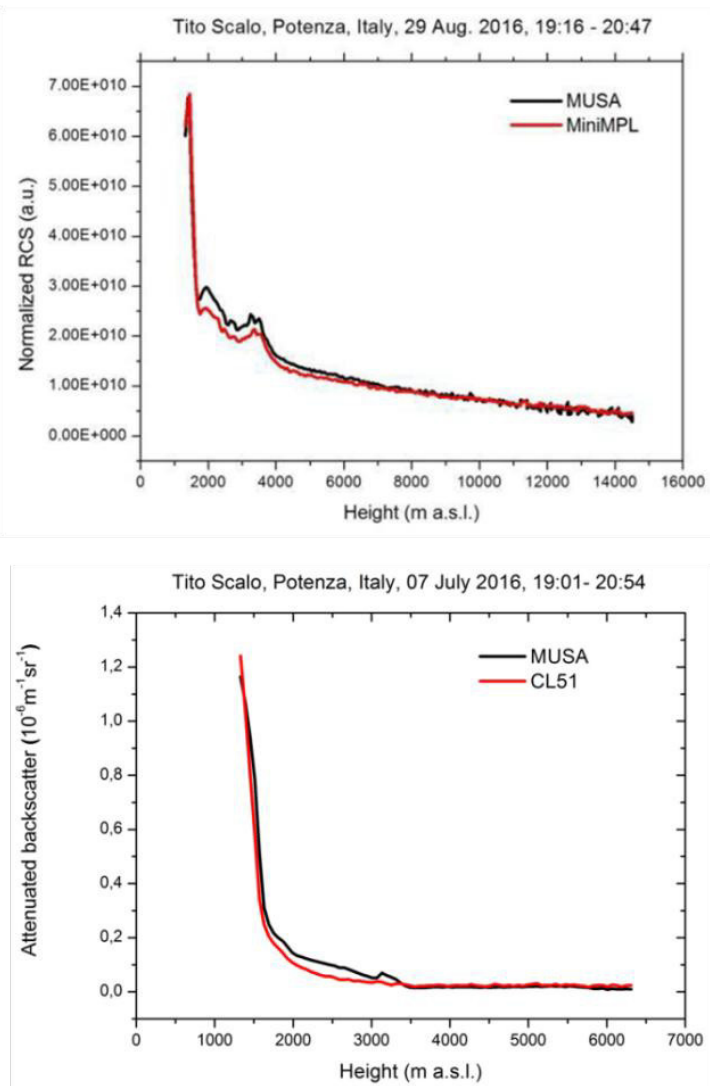

Figure 1. Comparison between range corrected signals (RCS) provided by MUSA and mini-MPL on 29 August 2016 in the time interval from 19:16 to 20:47UT (top panel) and attenuated backscatter coefficient profiles provided by MUSA and CL51 on 07 July 2016 in the time interval from 19:01 to 20:54 UT (bottom panel).

In the bottom panel of Figure 1, a typical comparison between the attenuated backscatter coefficient profiles of MUSA and CL51 is also reported. This refers to the observations of 7 July 2016 from 19:01 to 20:54 UT. The quicklooks of RCS time series (not reported) show the nocturnal boundary layer structure and above the presence of a residual layer up to an altitude higher than 3 $\mathrm{km}$ a.s.l. MUSA and CL51 profiles show that CL51 underestimates the attenuated backscatter 
coefficient value through the troposphere up to an altitude of about $3.4 \mathrm{~km}$ a.s.l. The differences are lower than $50 \%$ and they are probably due to a poor SNR of the ceilometer. The compared profiles also show different shapes in the investigated atmospheric region.

\section{RESULTS}

A first statistical analysis of simultaneous observations of all the instruments during the first half of the inter-comparison period, from July to September, is performed to investigate the differences of the mini-MPL and ceilometers with respect to MUSA. The top panel of Figure 2 shows the comparison of the frequency count distributions (fcds) of RCS values obtained from MUSA and mini-MPL. These distributions refer to 8 simultaneous measurement time series. The bottom panel of Figure 2 shows the fcds of attenuated backscatter coefficient values obtained from MUSA and CL51 and related to 13 simultaneous measurement time series. MUSA fcds are considered as the reference. The comparison of fcds confirms the overall good agreement between MUSA and mini-MPL, with some tendency of mini-MPL to overestimate MUSA for RCS values lower than $4.0 \times 10^{10}$, while for higher values mini-MPL underestimates MUSA. On the other hand, CL51 overestimates MUSA for the lowest values of attenuated backscatter coefficient, up to $2 \times 10^{-7} \mathrm{~m}^{-1} \mathrm{sr}^{-1}$, underestimates MUSA for values higher than $2 \mathrm{x}$ $10^{-7} \mathrm{~m}^{-1} \mathrm{sr}^{-1}$ up to $9 \times 10^{-7} \mathrm{~m}^{-1} \mathrm{sr}^{-1}$ and is in good agreement with MUSA for values higher than $9 \mathrm{x}$ $10^{-7} \mathrm{~m}^{-1} \mathrm{sr}^{-1}$. Moreover, fcds calculated in the vertical region above $4000 \mathrm{~m}$ a.s.l. (not reported) show that CL51 is not able to detect values of attenuated backscatter coefficient higher than $2 \mathrm{x}$ $10^{-7} \mathrm{~m}^{-1} \mathrm{sr}^{-1}$ at these altitude levels. The discrepancies between MUSA and CL51 are probably due to the ceilometer electronic distortions, reduced dymanic range, or low SNR, and not to calibration errors. Indeed, calibration errors should cause overestimations or underestimations of CL51 with respect to MUSA for all the values of fcds, though the effects of these errors might compensate over the whole dataset, so decreasing the discrepancies between fcds. In a different way, the ceilometer electronic distortions and low SNR can differently affect the different regions of fcds. These effects only partially depend on the water vapor absorption at the ceilometer wavelength, whose influence has been quantified by co-located Raman lidar and radiosounding water vapor measurements as input to radiative transfer models.
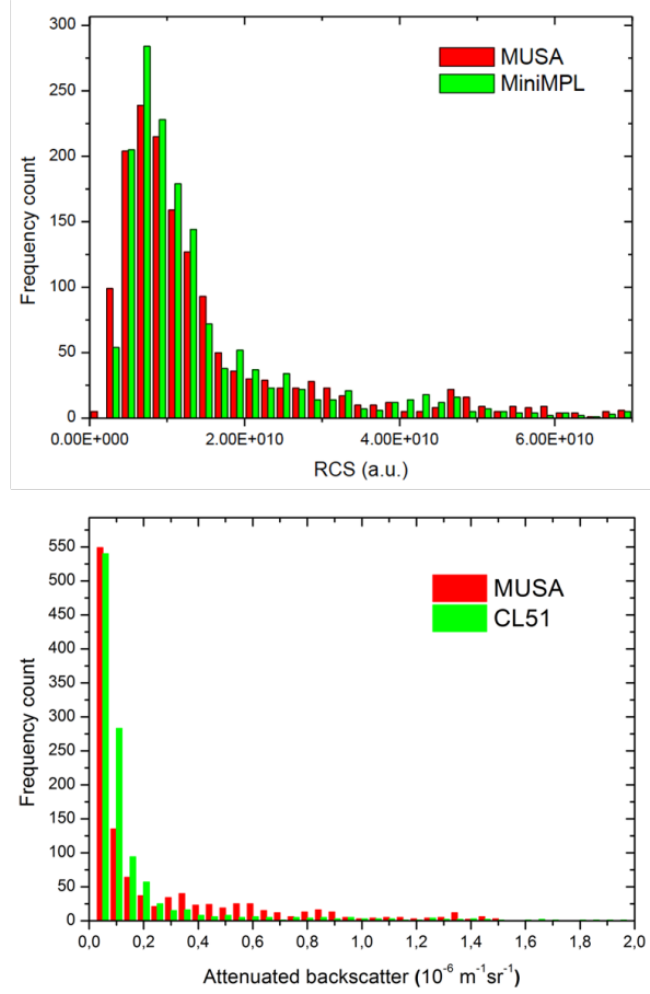

Figure 2. Frequency count distributions of RCS values retrieved from simultaneous observations of MUSA and mini-MPL (top panel) and attenuated backscatter coefficient values retrieved from simultaneous observations of MUSA and CL51 (bottom panel).

Figure 3 shows the profiles of the average percentage difference between MUSA and miniMPL values of RCS (top panel) and between MUSA and CL51 values of attenuated backscatter coefficient (bottom panel). The vertical bars are the standard deviations of average percentage differences. The profiles show that mini-MPL underestimates MUSA below the normalization region, with differences limited to $10 \%$. The variability of these differences ranges from \pm 10 $15 \%$ in the lower troposphere to about $\pm 5 \%$ in the free troposphere (up to $8000 \mathrm{~m}$ a.s.1.). This also confirms the good stability of mini-MPL in its operation in the considered time period. With the exception of a small vertical region, up to about $1500 \mathrm{~m}$ a.s.1., where CL51 overestimates MUSA with differences lower than of $40 \%$, and some 
uncertain spikes, CL51 underestimates MUSA up to an altitude of about $3700 \mathrm{~m}$ a.s.l., with maximum percentage differences around 50\%, probably due to the low SNR of CL51. Up to an altitude of about $2500 \mathrm{~m}$ a.s.l., differences are limited to $30 \%$. Above $3700 \mathrm{~m}$ a.s.l., CL51 overestimates MUSA, with differences oscillating around $50 \%$ up to an altitude of about $5000 \mathrm{~m}$ a.s.l. and drastically increasing at higher altitudes. These overestimations are likely due to the low SNR of CL51 and the large variability of these differences reveals also some instability of the ceilometer in the considered time period.
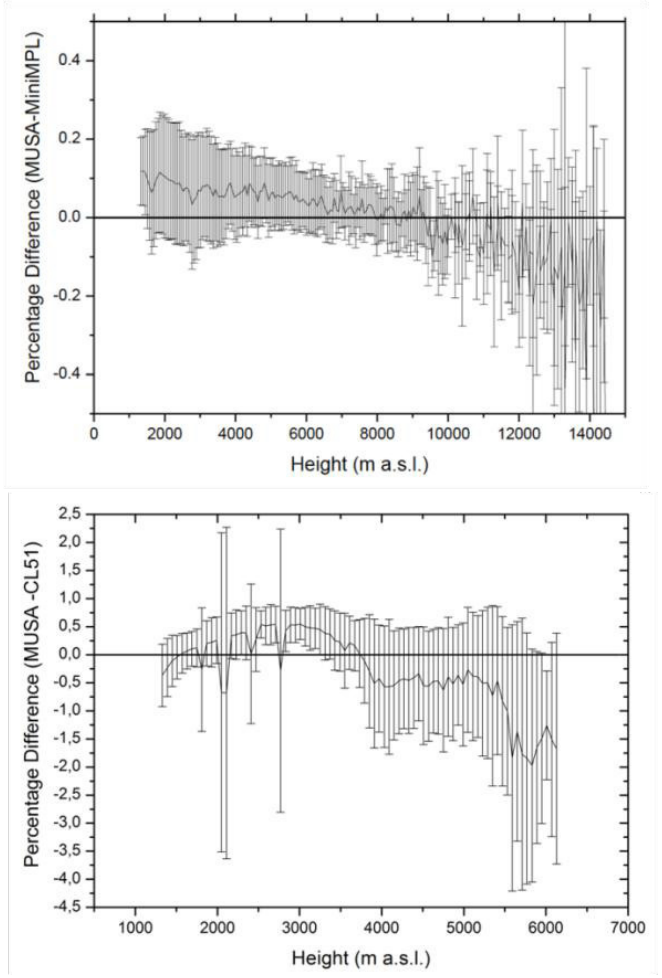

Figure 3. Profiles of the average percentage difference between MUSA and mini-MPL values of RCS (top panel) and MUSA and CL51 values of attenuated backscatter coefficient (bottom panel). The vertical bars are the standard deviations of average percentage differences.

\section{CONCLUSIONS}

A first statistical analysis of simultaneous observations of all the instruments involved in INTERACT - II during the first half of the intercomparison period is performed. This analysis reveals that, among the considered devices, the mini-MPL shows the best performances with discrepancies limited to $10 \%$ throughout the troposphere. In agreement with the outcome of INTERACT-I, ceilometers have fairly good performances in aerosol profiling in the lower troposphere, up to an altitude of about $2000 \mathrm{~m}$ above the ground, but they are limited at higher altitudes. The above preliminary analysis will be consolidated analyzing the cases collected in the period from October to December, including also the Lufft ceilometers data, and comparing miniMPL and MUSA profiles of aerosol optical properties. Further analysis is ongoing also to assess the stability of the considered lidar technologies with respect to variation of working and environment temperature, aerosol loading and laser operations. Finally, the results of INTERACT-II will be also compared to those reported in literature from other international experiments carried out to assess the ceilometer performances for aerosol profiling.

\section{ACKNOWLEDGEMENTS}

The financial support by the European Commission for ACTRIS-2 in HORIZON 2020 research and innovation programme under grant agreement no. 654109 is gratefully acknowledged. The authors would also like to thank Sigma Space Corporation, Vaisala and Campbell Scientific for contributing the mini-MPL, CL51 and CS135, respectively, for INTERACT - II campaign.

\section{References}

[1] D'Amico, G., et al., 2016: EARLINET Single Calculus Chain - technical Part 1: Preprocessing of raw lidar data, Atmos. Meas. Tech., 7, 1979-1997.

[2] Mattis, I., et al., 2016: EARLINET Single Calculus Chain - technical Part 2: Calculation of optical products, Atmos. Meas. Tech., 9, 3009-3029.

[3] Freudenthaler, V., et al., 2009: Depolarization ratio profiling at several wavelengths in pure Saharan dust during SAMUM 2006, Tellus B, $61,165-179$.

[4] Flynn, C.J., et al., 2007: Novel polarizationsensitive micropulse lidar measurement technique, Optics Express 15, 6,2785-2790.

[5] Madonna, F., et al., 2015: Ceilometer aerosol profiling versus Raman lidar in the frame of the INTERACT campaign of ACTRIS, Atmos. Meas. Tech., 8, 2207-2223. 